\title{
Time-triggered stochastic hybrid systems with two timer-dependent resets
}

\author{
Zahra Vahdat ${ }^{1}$, Zikai Xu${ }^{1}$ and Abhyudai Singh ${ }^{2 *}$
}

\begin{abstract}
We analyze a class of time-triggered stochastic hybrid systems where the state-space evolves as per a linear time-invariant dynamical system. This continuous time evolution is interspersed with two kinds of stochastic resets. The first reset occurs based on an internal timer that measures the time elapsed since it last occurred. Whenever the first reset occurs the states-space undergoes a random jump and the timer is reset to zero. The second reset occurs based on an arbitrary timer-depended rate, and whenever this reset fires, the state-space is changed based on a given random map. For this class of systems, we provide exact conditions that lead to finite statistic moments, and the corresponding exact analytical expressions for the first two moments. This framework is applied to study random fluctuations in the concentration of a protein in a growing cell. In the context of this example, the timer denotes the time elapsed since the cell was born, and the cell division event (first reset) is triggered based on a timer-dependent rate. The second reset corresponds to synthesis of the protein in stochastic bursts, and finally, during cell growth protein concentration continuously decrease due to dilution. Our analysis provides closed-form formulas for the noise in the protein concentration and leads to a striking result - for a constant (timer-independent) protein synthesis rate the noise in the protein concentration is invariant of the noise in the cell-cycle time. Finally, we provide a rigorous framework for investigating protein noise levels for different forms of time-dependent synthesis rates, as is the case for cell-cycle regulated genes inside the cell.
\end{abstract}

\section{Introduction}

Stochastic Hybrid Systems (SHS) are increasing being used to model dynamic random phenomena in a variety of engineering [1-9], biological [10-18], and

\footnotetext{
${ }^{* 1}$ Z. Vahdat and Z. Xu are with the Department of Electrical and Computer Engineering, University of Delaware, Newark, DE USA 19716. zahravd@udel.edu, zikai@udel.edu

$\dagger^{\dagger} \mathrm{A}$. Singh is with the Department of Electrical and Computer Engineering, Biomedical Engineering, Mathematical Sciences, Center for Bioinformatics and Computational Biology, University of Delaware, Newark, DE USA 19716. absingh@udel.edu
} 
physical systems [19]. Recent works have introduced Time-Triggered Stochastic Hybrid Systems where the state evolves as per a linear dynamical system. This continuous time evolution is interspersed by stochastic events that occur based on an internal timer. The timer measures the time elapsed since the last event, and creates memory in event timing. Whenever the event occurs the state undergoes a random jump based on a given random map, and the timer is reset to zero. These class of SHS have been shown to be quite useful in modeling and analysis of networked control systems [20-23].

A key contribution of this work is to generalize Time-Triggered SHS to include a second family of random resets (Fig. 1). This second family of resets occur based on a timer-dependent rate, and similar to the first family of reset, the state undergoes a stochastic jump whenever the corresponding events occur. While the first family of resets reinitializes the timer back to zero, the second family of resets has no effect on the timer. Note that these stochastic systems are essentially a subclass of Piecewise-deterministic Markov processes.

A second contribution of this work is to use this framework for capturing random fluctuations in the concentration of a protein inside a cell that undergoes periods of growth followed by division into two daughters. Advances in single-cell technologies over the last decade have dramatically captured fluctuations in protein levels within cells across organisms [24-32]. These random fluctuations have been shown to effect functioning of intracellular biomolecular circuits in both detrimental and beneficial ways [33-41]. Modeling frameworks that integrate different physiological noise mechanisms to predict the extent of protein level fluctuations as a function of experimentally tunable/measurable parameters are critically needed. Through our analysis we predict for the first time exact analytical formulas for the protein mean and noise levels, when the protein synthesis rate varies arbitrarily along the cell-cycle (i.e. as a function of $\boldsymbol{\tau})$ giving novel results and insights that can be tested with further experiments.

This paper is organized as follows. In Section 2 we provide the mathematical formulation of the stochastic system, and show its applicability in modeling protein concentration fluctuations inside an individual cell. In Section 3, we derive analytical formulas for the first and second order moments for the general SHS system with two timer-dependent resets. These results are applied to the protein synthesis example in Section 4, followed by conclusions in Section 5 . Before starting with the model formulation, we briefly discuss notation used throughout the paper.

Notation: $\mathbb{R}$ denotes the set of real numbers. We use hat to indicate constant vectors, e.g. $\hat{a}$, and matrices are denoted by capital letters. $A^{\top}$ is transpose of matrix $A$. $I_{n}$ is $n \times n$ identity matrix. We use small bold letters to denote random processes. $\langle\boldsymbol{x}\rangle$ is expected value of random process $\boldsymbol{x} . \overline{\langle\boldsymbol{x}\rangle} \equiv \lim _{t \rightarrow \infty}\langle\boldsymbol{x}\rangle$ is the expected value at the steady-state. $\langle\boldsymbol{x} \mid \boldsymbol{y}\rangle$ is the conditional expectation of $\boldsymbol{x}$ given another random variable $\boldsymbol{y}$. 


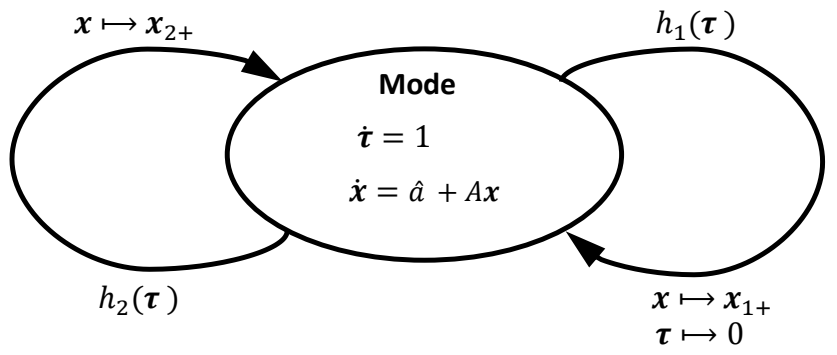

Figure 1: Model schematic of a stochastic hybrid system with two timerdependent resets. The state $\boldsymbol{x} \in \mathbb{R}^{n \times 1}$ evolves as per a linear time-invariant system and two family of resets occur at random times with rates $h_{i}(\boldsymbol{\tau}), i \in\{1,2\}$. Here $\boldsymbol{\tau}$ is a timer that measures the time elapsed since the last event belonging to the first family of resets. Whenever the events occur the state is reset as per $\boldsymbol{x} \mapsto \boldsymbol{x}_{i+}$, where $\boldsymbol{x}_{i+}$ is a random variable whose statistics is given by (9) and (10). The first family of resets also reinitializes the timer back to zero, while the second family of resets has no affect on the timer.

\section{Model formulation and biology example}

\subsection{Model Formulation}

The stochastic dynamical system is formulated as follows:

\subsubsection{Continuous dynamics}

The states of the SHS $\boldsymbol{x} \in \mathbb{R}^{n \times 1}$ evolve as per a linear time-invariant system

$$
\dot{\boldsymbol{x}}(t)=A \boldsymbol{x}+\hat{a},
$$

where $A \in \mathbb{R}^{n \times n}$ and $\hat{a} \in \mathbb{R}^{n \times 1}$ are constant matrix and vector, respectively. The continuous time evolution of the state space is interspersed by two families of random resets.

\subsubsection{The first family of resets}

The first family of resets is assumed to occur at times $\boldsymbol{t}_{s}, s \in\{1,2,3, \ldots\}$, such that the time intervals $\boldsymbol{\tau}_{\boldsymbol{s}} \equiv \boldsymbol{t}_{\boldsymbol{s}}-\boldsymbol{t}_{s-1}$ are independent and identically distributed random variables following an arbitrary positively-valued continuous probability density function (pdf) $f$. To model the timing of these resets, we introduce a timer $\boldsymbol{\tau}$ that linearly increases over time

$$
\dot{\tau}=1
$$

and resets to zero

$$
\tau \mapsto 0
$$


whenever a random event occurs. The occurrence of the next event depends on the state of the timer introducing memory in the event-arrival process. More specifically, the probability that an event occurs in the next infinitesimal time interval $(t, t+d t]$ is given by $h_{1}(\tau) d t$, where the hazard rate is

$$
h_{1}(\tau) \equiv \frac{f(\tau)}{1-\int_{y=0}^{\tau} f(y) d y} .
$$

As an example, if $f$ is exponentially-distributed with mean $\left\langle\boldsymbol{\tau}_{\boldsymbol{s}}\right\rangle$, then $h_{1}(\tau)=$ $1 /\left\langle\boldsymbol{\tau}_{\boldsymbol{s}}\right\rangle$ would be a constant corresponding to Poisson arrival of events. Defining the arrival of events as per (4) ensures that $\boldsymbol{\tau}_{\boldsymbol{s}}$ follows the pdf $f$

$$
\boldsymbol{\tau}_{\boldsymbol{s}} \sim f(\tau)=h_{1}(\tau) e^{-\int_{y=0}^{\tau} h_{1}(y) d y},
$$

and the corresponding pdf of $\boldsymbol{\tau}$ is given by

$$
\boldsymbol{\tau} \sim p(\tau)=\frac{1}{\left\langle\boldsymbol{\tau}_{\boldsymbol{s}}\right\rangle} e^{-\int_{y=0}^{\tau} h_{1}(y) d y}
$$

[42]. Moreover, the uncentered statistical moments of $\boldsymbol{\tau}$ are related to moments of $\boldsymbol{\tau}_{\boldsymbol{s}}$ as per

$$
\left\langle\boldsymbol{\tau}^{n}\right\rangle=\frac{\left\langle\boldsymbol{\tau}_{\boldsymbol{s}}{ }^{(n+1)}\right\rangle}{(n+1)\left\langle\boldsymbol{\tau}_{\boldsymbol{s}}\right\rangle} .
$$

Having modeled the timing of the first family of resets, we next describe its impact on the SHS state space. Each time this event occurs, the state of the system undergoes a random jump as per the reset

$$
\boldsymbol{x} \mapsto \boldsymbol{x}_{1^{+}}, \boldsymbol{\tau} \mapsto 0,
$$

where $\boldsymbol{x}_{1}^{+}$represents the state of the system immediately after the event belonging to the first family of resets. We assume $\boldsymbol{x}_{1}$ to be a random variable whose statistics depend on the value of $\boldsymbol{x}$ just before the event. More specifically, the average value of $\boldsymbol{x}_{1}^{+}$is

$$
\left\langle\boldsymbol{x}_{1+}\right\rangle=J_{1} \boldsymbol{x}+\hat{r}_{1},
$$

where $J_{1} \in \mathbb{R}^{n \times n}$ and $\hat{r}_{1} \in \mathbb{R}^{n \times 1}$ are constant matrix and vector, respectively. Furthermore, the covariance matrix $\boldsymbol{x}_{1}^{+}$is given as

$$
\begin{aligned}
& \left\langle\boldsymbol{x}_{1+} \boldsymbol{x}_{1+}^{\top}\right\rangle-\left\langle\boldsymbol{x}_{1+}\right\rangle\left\langle\boldsymbol{x}_{1+}^{\top}\right\rangle= \\
& Q_{1} \boldsymbol{x} \boldsymbol{x}^{\top} Q_{1}^{\top}+B_{1} \boldsymbol{x} \hat{c}_{1}^{\top}+\hat{c}_{1} \boldsymbol{x} B_{1}^{\top}+G_{1},
\end{aligned}
$$

where $Q_{1} \in \mathbb{R}^{n \times n}, B_{1} \in \mathbb{R}^{n \times n}$ are constant matrices, $G_{1} \in \mathbb{R}^{n \times n}$ is a constant symmetric positive semi-definite matrix, and $\hat{c}_{1} \in \mathbb{R}^{n \times 1}$ is a constant vector. In essence, (10) represents the noise added when the event is triggered, and this noise can be state-dependent. 


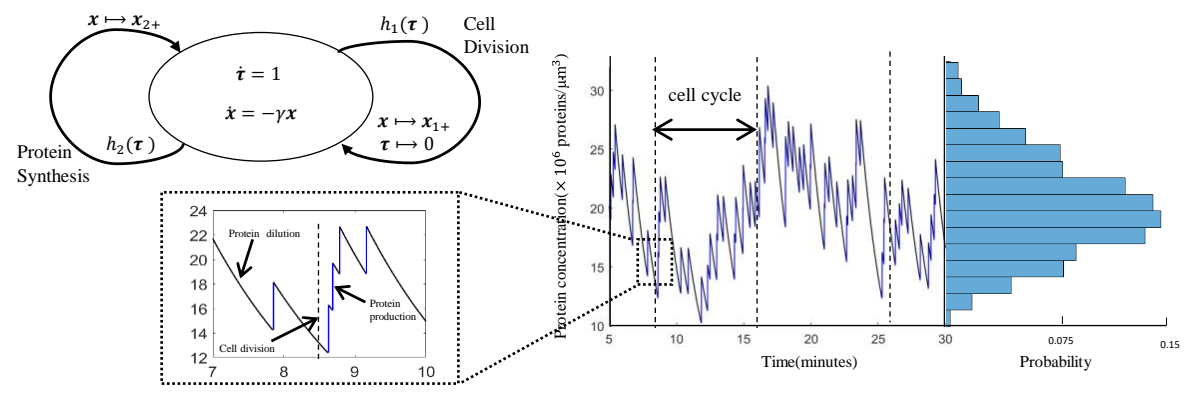

Figure 2: Modeling protein concentration in a single cell using timetriggered stochastic hybrid system: Right: Protein level $\boldsymbol{x}$ is modeled via a SHS with two families of stochastic resets. The first family of resets represent cell-division events that occur randomly with rate $h_{1}(\boldsymbol{\tau})$ where $\boldsymbol{\tau}$ is cell-cycle timer measuring time elapsed since the cell was born. Whenever the cell divides, the protein level changes as per (15) and (16). The second family of resets correspond to production of proteins in bursts of size $\boldsymbol{u}$, and these burst events occur with rate $h_{2}(\boldsymbol{\tau})$. For each burst event, the protein level changes as per (17) and (18). Left: Sample realization of the protein concentration showing increase due to burst events, exponential decay between two burst events due to dilution, and cell division events that do not change the mean concentration but add partitioning noise.

\subsubsection{The second family of resets}

These resets occur randomly at a timer-dependent rate $h_{2}(\boldsymbol{\tau})$, where $h_{2}$ is an arbitrary positive-valued function, i.e., the probability that the second family of reset happens in the next infinitesimal time $(t, t+d t]$ is $h_{2}(\boldsymbol{\tau}) d t$. Whenever these resets occur the state is reset as

$$
\boldsymbol{x} \mapsto \boldsymbol{x}_{2^{+}},
$$

where $\boldsymbol{x}_{2+}$ denotes the state of the system just after the event belonging to the second family of resets. Similar to (8), the average jump in $\boldsymbol{x}_{2^{+}}$is

$$
\left\langle\boldsymbol{x}_{2^{+}}\right\rangle=J_{2} \boldsymbol{x}+\hat{r}_{2},
$$

where $J_{2} \in \mathbb{R}^{n \times n}$ and $\hat{r}_{2} \in \mathbb{R}^{n \times 1}$ are constant matrix and vector, respectively. Furthermore, the covariance matrix of $\boldsymbol{x}_{2^{+}}$is same as (10) by replacing $Q_{2}, B_{2}$, $\hat{c}_{2}$ and $G_{2}$ with $Q_{1}, B_{1}, \hat{c}_{1}$ and $G_{1}$ respectively. It is important to point out that unlike the first family of resets, the second family of resets do not affect the timer. While the occurrences of all stochastic events are timer driven, only events of the first family of resets reinitialize the timer back to zero (Fig. 1).

\subsection{Biology Example}

It turns out that the above SHS framework with two-timer dependent resets is ideal for capturing stochastic fluctuations in the concentration of a protein 
within growing cells. Before proceeding with a rigorous analysis of the SHS, we provide some details on this example.

Consider a newborn cell, whose volume grows exponentially over time with rate $\gamma$, and after a period of growth, the cell symmetrically divides into two daughters. These randomly-timed cell division events are the first family of resets. Here the timer $\boldsymbol{\tau}$ denotes the time elapsed since the birth of cell, and the next cell division event is assuming to occur with rate $h_{1}(\boldsymbol{\tau})$. Choosing this rate as in (4) ensures that the time duration from cell birth to division $\boldsymbol{\tau}_{\boldsymbol{s}}$ follows a prior probability distribution $f$. Let scalar $\boldsymbol{x}(t)$ denote the concentration of a protein. Then during cell growth, the protein level is continuously diluted as

$$
\dot{\boldsymbol{x}}(t)=-\gamma \boldsymbol{x} .
$$

where the growth rate is related to the average cell-cycle duration

$$
\left\langle\boldsymbol{\tau}_{\boldsymbol{s}}\right\rangle=\frac{\ln 2}{\gamma} .
$$

This implies $A=-\gamma, \hat{a}=0$ in (1). Note that a cell division event does not change the concentration of a protein (both the cell volume and the protein copy number are approximately halved), but it introduces some noise due to errors from random partitioning of protein molecules between two daughters [43-45]. Towards that end, the reset map for the first family of rests is given by

$$
\left\langle\boldsymbol{x}_{1+}\right\rangle=\boldsymbol{x} .
$$

and

$$
\left\langle\boldsymbol{x}_{1+}^{2}\right\rangle-\left\langle\boldsymbol{x}_{1^{+}}\right\rangle^{2}=b \boldsymbol{x},
$$

where the parameter $b$ quantifies the extent of partitioning noise [43]. Comparing (15) and (16) with (9) and (10) leads to the following parameters for the first family resets: $J_{1}=1, B_{1}=b / 2, \hat{c}_{1}=1$ and $\hat{r}_{1}=Q_{1}=G_{1}=0$.

The second family of resets correspond to the stochastic synthesis of a protein in bursts as has been shown experimentally [24,46-50], and modeled previously [51-56]. In particular, the burst events arrive at rate $h_{1}(\boldsymbol{\tau})$ that can depend on the cell-cycle timer. Whenever the burst event occurs, the protein concentration increases by a burst size $\boldsymbol{u}$, where $\boldsymbol{u}$ is assumed to be an identically and independently distributed random variable. Based on this formulation of the second family of resets

$$
\left\langle\boldsymbol{x}_{2^{+}}\right\rangle=\boldsymbol{x}+\langle\boldsymbol{u}\rangle
$$

and

$$
\left\langle\boldsymbol{x}_{2^{+}}^{2}\right\rangle-\left\langle\boldsymbol{x}_{2^{+}}\right\rangle^{2}=\left\langle\boldsymbol{u}^{2}\right\rangle-\langle\boldsymbol{u}\rangle^{2},
$$

are implying $J_{2}=1, \hat{r}_{2}=\langle\boldsymbol{u}\rangle, Q_{2}=B_{2}=\hat{c}_{2}=0$ and $G_{2}=\left\langle\boldsymbol{u}^{2}\right\rangle-\langle\boldsymbol{u}\rangle^{2}$. In summary, the SHS framework allows integration of three distinct noise mechanisms critically affecting gene expression: synthesis of a protein in random bursts, the division of a cell into two daughters at random times, and randomness in the partitioning of a protein between daughters. A typical stochastic realization of this SHS model is illustrated in Fig. 2. 


\section{$3 \quad$ statistical moments}

Having formulated the SHS with two-timer dependent resets, we next derive the statistical moments of $\boldsymbol{x}$.

\subsection{The first-order moment}

We start by outlining our approach and then summarize the main result in Theorem 1. In between two successive events of the first family of resets, the conditional mean $\langle\boldsymbol{x} \mid \boldsymbol{\tau}\rangle$ evolves as follows (see Appendix A for details)

$$
\frac{\partial\langle\boldsymbol{x} \mid \boldsymbol{\tau}\rangle}{\partial \tau}=A_{x}(\tau)\langle\boldsymbol{x} \mid \boldsymbol{\tau}\rangle+\hat{a}_{x}(\tau)
$$

where

$$
\begin{aligned}
& A_{x}(\tau)=A+h_{2}(\tau)\left(J_{2}-I_{n}\right), \\
& \hat{a}_{x}(\tau)=\hat{a}+h_{2}(\tau) \hat{r}_{2} .
\end{aligned}
$$

This time variant system leads to the following conditional mean

$$
\begin{aligned}
\langle\boldsymbol{x} \mid \boldsymbol{\tau}=\tau\rangle & =e^{\int_{0}^{\tau} A_{x}(y) d y}\langle\boldsymbol{x} \mid \boldsymbol{\tau}=0\rangle \\
& +e^{\int_{0}^{\tau} A_{x}(y) d y} \int_{0}^{\tau} e^{-\int_{0}^{l} A_{x}(y) d y} \hat{a}_{x}(l) d l,
\end{aligned}
$$

where $\langle\boldsymbol{x} \mid \boldsymbol{\tau}=0\rangle$ is the expected value just after an event of the first family of resets. In order to calculate $\overline{\langle\boldsymbol{x} \mid \boldsymbol{\tau}=0\rangle}$, we apply (9)

$$
\overline{\langle\boldsymbol{x} \mid \boldsymbol{\tau}=0\rangle}=J_{1} \overline{\left\langle\boldsymbol{x} \mid \boldsymbol{\tau}=\boldsymbol{\tau}_{\boldsymbol{s}}\right\rangle}+\hat{r}_{1},
$$

and then substituting $\left\langle\boldsymbol{x} \mid \boldsymbol{\tau}=\boldsymbol{\tau}_{\boldsymbol{s}}\right\rangle$ from (21) yields

$$
\begin{aligned}
\overline{\langle\boldsymbol{x} \mid \boldsymbol{\tau}=0\rangle} & =\left(I_{n}-J_{1}\left\langle e^{\int_{0}^{\boldsymbol{\tau}_{\boldsymbol{s}}} A_{x}(y) d y}\right\rangle\right)^{-1} \times \\
& \left(J_{1}\left\langle e^{\int_{0}^{\boldsymbol{\tau}_{\boldsymbol{s}}} A_{x}(y) d y} \int_{0}^{\boldsymbol{\tau}_{\boldsymbol{s}}} e^{-\int_{0}^{l} A_{x}(y) d y} \hat{a}_{x}(l) d l\right\rangle+\hat{r}_{1}\right) .
\end{aligned}
$$

Once we have solved for $\langle\boldsymbol{x} \mid \boldsymbol{\tau}=0\rangle$, we obtain $\langle\boldsymbol{x} \mid \boldsymbol{\tau}=\tau\rangle$ from (21). Finally, we use (6) to uncondition $\langle\boldsymbol{x} \mid \boldsymbol{\tau}=\tau\rangle$ with respect to $\boldsymbol{\tau}$ to get the expected value of $\boldsymbol{x}$. This result can be formally summarized as follows.

Theorem 1 Consider the SHS with two-timer dependent resets as formulated in (1)-(12). If all eigenvalues of $J_{1}\left\langle e^{\int_{0}^{\tau_{s}} A_{x}(y) d y}\right\rangle$ are inside the unit circle, then the first-order moment exists and is given by

$$
\begin{aligned}
& \overline{\langle\boldsymbol{x}\rangle}=\left\langle e^{\int_{0}^{\boldsymbol{\tau}} A_{x}(y) d y}\right\rangle\left(I_{n}-J_{1}\left\langle e^{\int_{0}^{\boldsymbol{\tau}_{s}} A_{x}(y) d y}\right\rangle\right)^{-1} \times \\
& \left(J_{1}\left\langle e^{\int_{0}^{\boldsymbol{\tau}_{\boldsymbol{s}}} A_{x}(y) d y} \int_{0}^{\boldsymbol{\tau}_{\boldsymbol{s}}} e^{-\int_{0}^{l} A_{x}(y) d y} \hat{a}_{x}(l) d l\right\rangle+\hat{r}_{1}\right) \\
& +\left\langle e^{\int_{0}^{\boldsymbol{\tau}} A_{x}(y) d y} \int_{0}^{\boldsymbol{\tau}} e^{-\int_{0}^{l} A_{x}(y) d y} \hat{a}_{x}(l) d l\right\rangle
\end{aligned}
$$

where

$$
\begin{aligned}
& A_{x}(y)=A+h_{2}(y)\left(J_{2}-I_{n}\right), \\
& \hat{a}_{x}(y)=\hat{a}+h_{2}(y) \hat{r}_{2} .
\end{aligned}
$$


A special case of the Theorem is when $h_{2}(\boldsymbol{\tau})=0$ and the system reduces to a single family of resets as considered in [42]. For completeness we provide this result as a Corollary.

Corollary 1 Assume that the system is described by (1)-(12) with $h_{2}(\boldsymbol{\tau})=0$ and all eigenvalues of the matrix $J_{1}\left\langle e^{A \tau_{s}}\right\rangle$ are inside the unit circle. Then the first order moment of the system is

$$
\begin{aligned}
\overline{\langle\boldsymbol{x}\rangle} & =\left\langle e^{A \boldsymbol{\tau}}\right\rangle\left(I_{n}-J_{1}\left\langle e^{A \tau_{s}}\right\rangle\right)^{-1}\left(J_{1}\left\langle e^{A \tau_{s}} \int_{0}^{\tau_{s}} e^{-A l} \hat{a} d l\right\rangle\right. \\
& \left.+\hat{r}_{1}\right)+\left\langle e^{A \boldsymbol{\tau}} \int_{0}^{\tau} e^{-A l} \hat{a} d l\right\rangle .
\end{aligned}
$$

See the details for computing $\left\langle e^{A \boldsymbol{\tau}}\right\rangle$ and $\left\langle e^{A \boldsymbol{\tau}_{s}} \int_{0}^{\boldsymbol{\tau}_{s}} e^{-A l} \hat{a} d l\right\rangle$ in [18].

\subsection{Second-order moments}

To derive the second-order moment we consider the time evolution of the conditioned covariance matrix $\left\langle\boldsymbol{x} \boldsymbol{x}^{\top} \mid \boldsymbol{\tau}\right\rangle$

$$
\frac{\partial\left\langle\boldsymbol{x} \boldsymbol{x}^{\top} \mid \boldsymbol{\tau}\right\rangle}{\partial t}=A \boldsymbol{x} \boldsymbol{x}^{\top}+\boldsymbol{x} \boldsymbol{x}^{\top} A^{\top}+\hat{a} \boldsymbol{x}^{\top}+\boldsymbol{x} \hat{a}^{\top} .
$$

By vectorization, we replace $\boldsymbol{x} \boldsymbol{x}^{\top}$ with $\operatorname{vec}\left(\boldsymbol{x} \boldsymbol{x}^{\top}\right)$. Defining

$$
\boldsymbol{\mu} \equiv\left[\boldsymbol{x}^{\top} \operatorname{vec}\left(\boldsymbol{x} \boldsymbol{x}^{\top}\right)^{\top}\right]^{\top},
$$

then the continuous dynamics of $\boldsymbol{\mu}$ follows

$$
\dot{\boldsymbol{\mu}}=A_{\mu} \boldsymbol{\mu}+\hat{a}_{\mu},
$$

where

$$
\begin{aligned}
A_{\mu} & =\left[\begin{array}{c:c}
A & 0 \\
\hdashline I_{n} \otimes \hat{a}+\hat{a} \otimes I_{n} & I_{n} \otimes A+A \otimes I_{n}
\end{array}\right], \\
\hat{a}_{\mu} & =\left[\begin{array}{c}
\hat{a} \\
\hdashline 0
\end{array}\right],
\end{aligned}
$$

and $\otimes$ stands the Kronecker Product [42]. When the first family $(i=1)$ or the second family $(i=2)$ of resets occurs, $\boldsymbol{\mu}$ changes as per the map

$$
\boldsymbol{\mu}_{i^{+}}=J_{\mu_{i}} \boldsymbol{\mu}+\hat{r}_{\mu_{i}}, i \in\{1,2\},
$$

where

$$
\begin{gathered}
J_{\mu_{i}}=\left[\begin{array}{c:c}
J_{i} & 0 \\
\hdashline B_{i} \otimes \hat{c}_{i}+\hat{c}_{i} \otimes B_{i} & \\
+J_{i} \otimes \hat{r}_{i}+\hat{r}_{i} \otimes J_{i} & J_{i} \otimes J_{i}+Q_{i} \otimes Q_{i}
\end{array}\right], \\
\hat{r}_{\mu_{i}}=\left[\begin{array}{c:c}
\hat{r}_{i} \\
\hdashline \operatorname{vec}\left(\bar{G}_{i}+\hat{r}_{i} \hat{r}_{i}^{\top}\right)
\end{array}\right] .
\end{gathered}
$$

Having recast the stochastic dynamics of $\boldsymbol{\mu}$, also as a SHS with two family of resets, the expected value of $\boldsymbol{\mu}$ (and hence $\boldsymbol{x} \boldsymbol{x}^{\top}$ ) can be obtained by applying Theorem 1 on this augmented system. While the analysis presented here is restricted to the first and second order moments, a similar approach can be applied for deriving higher order moments. 


\section{Revisiting Stochastic Protein Synthesis}

Having derived the first two moments of $\boldsymbol{x}$ in the general setting, we now revisit the biological example introduced in Section II. Recall that in the example, the scalar $\boldsymbol{x}$ denote the concentration of a protein that is continuously diluted from cell growth via (13). The first family of resets represent cell-division events that reset the state as per (15) and (16). The second family of resets correspond to proteins bursts that reset the state as per as per (17) and (18).

Defining the vector $\boldsymbol{\mu} \equiv\left[\begin{array}{ll}\boldsymbol{x} & \boldsymbol{x}^{2}\end{array}\right]^{\top}$, then the continuous dynamics of $\boldsymbol{\mu}$ between resets is given by (29) with

$$
A_{\mu}=\left[\begin{array}{cc}
-\gamma & 0 \\
0 & -2 \gamma
\end{array}\right], \hat{a}_{\mu}=\left[\begin{array}{l}
0 \\
0
\end{array}\right]
$$

Using (31), the resets in $\boldsymbol{\mu}$ are

$$
J_{\mu_{1}}=\left[\begin{array}{ll}
1 & 0 \\
b & 1
\end{array}\right], \hat{r}_{\mu_{1}}=\left[\begin{array}{l}
0 \\
0
\end{array}\right]
$$

and

$$
J_{\mu_{2}}=\left[\begin{array}{cc}
1 & 0 \\
2\langle\boldsymbol{u}\rangle & 1
\end{array}\right], \hat{r}_{\mu_{2}}=\left[\begin{array}{c}
\langle\boldsymbol{u}\rangle \\
\left\langle\boldsymbol{u}^{2}\right\rangle
\end{array}\right]
$$

corresponding to the first and second families of resets, respectively. Applying Theorem 1 on this augmented system yields the first two steady-state moments of the protein concentration. As the formula for the second-order moment is quite lengthy, we only provide the result for the mean protein concentration that is given by

$$
\begin{aligned}
\overline{\langle\boldsymbol{x}\rangle} & =\frac{\langle\boldsymbol{u}\rangle}{\gamma\left\langle\boldsymbol{\tau}_{\boldsymbol{s}}\right\rangle}\left\langle e^{-\gamma \boldsymbol{\tau}_{\boldsymbol{s}}} \int_{0}^{\boldsymbol{\tau}_{\boldsymbol{s}}} e^{\gamma l} h_{2}(l) d l\right\rangle \\
& +\langle\boldsymbol{u}\rangle\left\langle e^{-\gamma \boldsymbol{\tau}} \int_{0}^{\boldsymbol{\tau}} e^{\gamma l} h_{2}(l) d l\right\rangle,
\end{aligned}
$$

with $\langle\boldsymbol{u}\rangle$ being the mean burst size, $\gamma$ the protein dilution rate, and $h_{2}(\boldsymbol{\tau})$ the timer-dependent protein synthesis rate. Next, we explore protein mean and noise levels for specific forms of the synthesis rate. We quantify protein noise levels by the steady-state Fano factor (variance/mean)

$$
F F \equiv \frac{\overline{\left\langle\boldsymbol{x}^{2}\right\rangle}-\overline{\langle\boldsymbol{x}\rangle^{2}}}{\overline{\langle\boldsymbol{x}\rangle}} .
$$

\subsection{Constant protein synthesis rate}

For a constant protein synthesis rate

$$
h_{2}(\boldsymbol{\tau})=k_{1},
$$

using (14) into (37) we get

$$
\overline{\langle\boldsymbol{x}\rangle}=\frac{k_{1}\langle\boldsymbol{u}\rangle\left\langle\boldsymbol{\tau}_{\boldsymbol{s}}\right\rangle}{\ln (2)}
$$


Moreover, the steady-state Fano factor of the protein concentration obtained as

$$
F F=\underbrace{\frac{\left\langle\boldsymbol{u}^{2}\right\rangle}{2\langle\boldsymbol{u}\rangle}}_{\text {Protein bursting noise }}+\underbrace{\frac{b}{2 \ln (2)}}_{\text {Partitioning noise }}
$$

can be decomposed into two components. The first component represents the contribution from stochastic bursting, and the second component arises due to partitioning errors at the time of cell division. It is remarkable that in this case the Fano factor is independent of $k_{1}, \gamma$ and the moments of the cell-cycle time $\tau_{s}$.

\subsection{Linearly increasing protein synthesis rate}

We next assume that the protein synthesis rate is a linear increasing function of $\tau$

$$
h_{2}(\boldsymbol{\tau})=k_{2} \boldsymbol{\tau}+k_{1},
$$

with positive constants $k_{1}$ and $k_{2}$. Then, using a similar procedure we obtain

$$
\overline{\langle\boldsymbol{x}\rangle}=\frac{k_{1}\langle\boldsymbol{u}\rangle\left\langle\boldsymbol{\tau}_{\boldsymbol{s}}\right\rangle}{\ln (2)}+\frac{k_{2}\langle\boldsymbol{u}\rangle\left(C V_{\boldsymbol{\tau}_{s}}^{2}+1\right)\left\langle\boldsymbol{\tau}_{\boldsymbol{s}}\right\rangle^{2}}{2 \ln (2)},
$$

which depends on the average cell-cycle duration $\left\langle\boldsymbol{\tau}_{\boldsymbol{s}}\right\rangle$, and interestingly, also depends on the noise in cell-cycle time $C V_{\boldsymbol{\tau}_{s}}^{2}$ as quantified by its coefficient of variation squared. The Fano factor of the protein concentration is given by (44) , and it can be decomposed into three components representing contributions from stochastic bursting, partitioning noise, and cell cycle noise

$$
\begin{aligned}
& F F=\underbrace{\frac{\left\langle\boldsymbol{u}^{2}\right\rangle}{2\langle\boldsymbol{u}\rangle}}_{\text {Protein bursting noise }}+ \\
& \underbrace{b\left(\frac{k_{2}\left\langle\boldsymbol{\tau}_{\boldsymbol{s}}\right\rangle\left(\left\langle e^{-\gamma \boldsymbol{\tau}_{\boldsymbol{s}}}\right\rangle-1+\ln (2)\right)}{\left(1-\left\langle e^{-\gamma \boldsymbol{\tau}_{\boldsymbol{s}}}\right\rangle\right) \ln ^{2}(2)\left(\left(C V_{\boldsymbol{\tau}_{\boldsymbol{s}}}^{2}+1\right) k_{2}\left\langle\boldsymbol{\tau}_{\boldsymbol{s}}\right\rangle+2 k_{1}\right)}+\frac{k_{1}}{\ln (2)\left(\left(C V_{\boldsymbol{\tau}_{\boldsymbol{s}}}^{2}+1\right) k_{2}\left\langle\boldsymbol{\tau}_{\boldsymbol{s}}\right\rangle+2 k_{1}\right)}\right)}_{\text {Partitioning noise }}+ \\
& \left(\langle \boldsymbol { u } \rangle k _ { 2 } { } ^ { 2 } \left(\left(1-\left\langle e^{-\gamma \boldsymbol{\tau}_{\boldsymbol{s}}}\right\rangle\right)\left\langle\boldsymbol{\tau}_{\boldsymbol{s}}\right\rangle^{3}\left(3\left(C V_{\boldsymbol{\tau}_{\boldsymbol{s}}}^{2}+1\right)^{2} \ln ^{2}(2)+6\left(C V_{\boldsymbol{\tau}_{\boldsymbol{s}}}^{2}+1\right) \ln (2)-12\right)+4\left(\left\langle e^{-\gamma \boldsymbol{\tau}_{\boldsymbol{s}}}\right\rangle\right.\right.\right. \\
& \left.\left.-1)\left\langle\boldsymbol{\tau}_{\boldsymbol{s}}{ }^{3}\right\rangle \ln ^{2}(2)+12\left\langle\boldsymbol{\tau}_{\boldsymbol{s}} e^{-\gamma \boldsymbol{\tau}_{\boldsymbol{s}}}\right\rangle\left\langle\boldsymbol{\tau}_{\boldsymbol{s}}\right\rangle^{2} \ln (2)\right)\right) \times \frac{1}{6\left(\left\langlee^{\left.\left.-\gamma \boldsymbol{\tau}_{\boldsymbol{s}}\right\rangle-1\right)} \ln ^{3}(2)\left(\left(C_{\boldsymbol{\tau}_{\boldsymbol{s}}}^{2}+1\right) k_{2}\left\langle\boldsymbol{\tau}_{\boldsymbol{s}}\right\rangle+2 k_{1}\right)\right.\right.} \\
& \text { Cell-cycle noise }
\end{aligned}
$$

To get some insight into how these components vary with $C V_{\boldsymbol{\tau}_{s}}^{2}$, we consider a Gamma-distributed $\boldsymbol{\tau}_{\boldsymbol{s}}$. For this purpose, the terms $\left\langle e^{-\gamma \boldsymbol{\tau}_{\boldsymbol{s}}}\right\rangle,\left\langle\boldsymbol{\tau}_{\boldsymbol{s}} e^{-\gamma \boldsymbol{\tau}_{\boldsymbol{s}}}\right\rangle$ and $\left\langle\boldsymbol{\tau}_{\boldsymbol{s}}{ }^{3}\right\rangle$ in (44) are further simplified and provided in Appendix B. In Fig. 3, we plot the three different noise components of Fano factor from (44) as a function of $C V_{\boldsymbol{\tau}_{s}}^{2}$ and observe contrasting behaviors - the noise contribution from bursting remains constant, the noise contribution from partitioning decreases, and finally, the noise contribution from cell-cycle noise increases. Fig. 4 plots the overall 


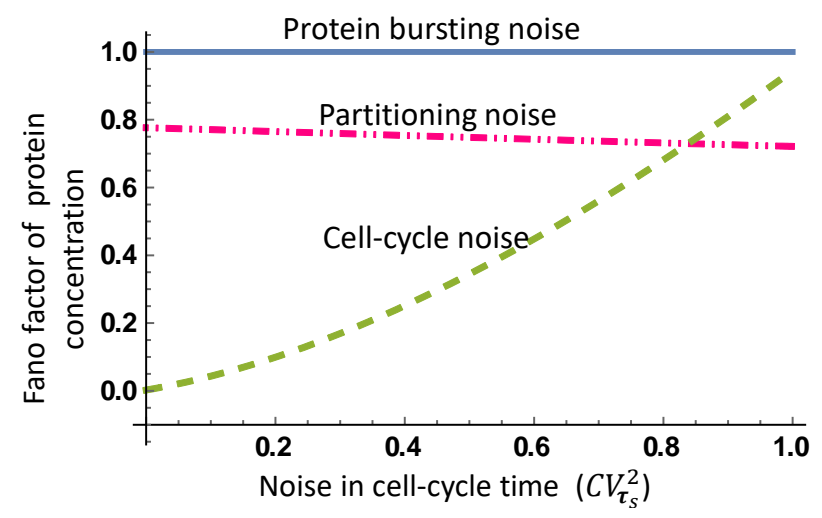

Figure 3: Protein noise components are affected differently by the noise in the cell-cycle time.. For a Gamma-distributed $\boldsymbol{\tau}_{\boldsymbol{s}}$, the noise components in (44) are plotted with respect to $C V_{\boldsymbol{\tau}_{s}}^{2}$. Interestingly contrasting behaviors emerge with the noise contribution from bursting remaining constant, the noise contribution from partitioning decreasing, and the noise contribution from cell-cycle noise increasing as $C V_{\tau_{s}}^{2}$ varies from zero (deterministic cell-cycle times) to one (exponentially distributed cell-cycle times). Other parameters taken as $\left\langle\boldsymbol{\tau}_{\boldsymbol{s}}\right\rangle=1$ hour, $\left\langle\boldsymbol{u}^{2}\right\rangle=2,\langle\boldsymbol{u}\rangle=1, b=1$, $k_{1}=1$ hour $^{-1}, k_{2}=2$ hour $^{-2}$.

Fano factor as a function of $C V_{\tau_{s}}^{2}$ and depending on the relative contributions, it can either remain constant, monotonically increase, or vary non-monotonically with $C V_{\tau_{s}}^{2}$.

\section{Conclusion}

We formulate a class of time-triggered stochastic hybrid systems with two families of resets, and exploited this framework to investigate noise in the concentration of a given protein. Our analysis provides the first results connecting the protein mean and noise levels to distinct noise mechanisms (stochastic bursting, noise in the cell-cycle time, and randomness in partitioning of molecules between two cells). A key highlight of our results is that for a constant protein production rate, the noise in the protein level becomes invariant of both the mean cell cycle time, and the noise in the cell cycle time. This natural buffering of protein noise levels to the cell cycle is intriguing, and can be experimentally tested by measuring protein noise levels for changing growth conditions in a given cell type.

When the protein production is timer-dependent we derive exact analytical formulas for the statistical moments of $\boldsymbol{x}$. In this case, the noise in the protein level can be decomposed into three components corresponding to the three different noise mechanisms. Our analysis shows that the noise component from bursting is invariant of $\boldsymbol{\tau}_{\boldsymbol{s}}$, the noise component for partitioning slightly decreases with increasing noise $C V_{\tau_{s}}^{2}$, and finally the noise contribution from 


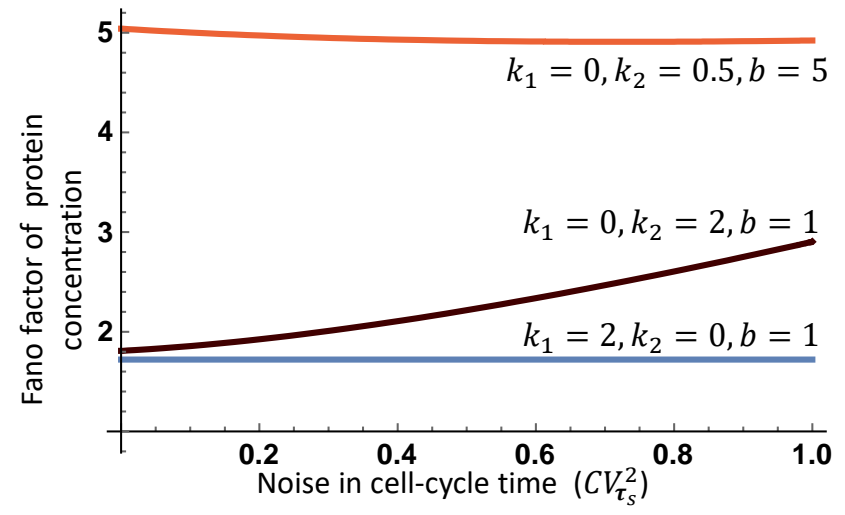

Figure 4: Depending on the protein synthesis rate and partitioning noise, noise in protein concentration may decrease, increase or remain invariant of the noise in the cell-cycle timing. Steady-state Fano factor of protein concentration as a function of the noise in the cell-cycle timing for a Gamma-distributed $\boldsymbol{\tau}_{\boldsymbol{s}}$ and synthesis rate (42). If the coefficient $k_{2}$ is zero, Fano factor of protein concentration remains constant with respect to changes in $C V_{\tau_{s}}^{2}$. For other values of $k_{2}$ and $k_{1}$, Fano factor may be an increasing or decreasing function of $C V_{\tau_{s}}^{2}$ depending on the value of $b$. Other parameters are taken as $\left\langle\boldsymbol{\tau}_{\boldsymbol{s}}\right\rangle=1$ hour $,\left\langle\boldsymbol{u}^{2}\right\rangle=2,\langle\boldsymbol{u}\rangle=1$.

cell cycle increases sharply with $C V_{\boldsymbol{\tau}_{s}}^{2}$. While the analysis here was restricted to a simple linear production rate $h_{2}(\boldsymbol{\tau})=k_{2} \boldsymbol{\tau}+k_{1}$, we plan to investigate more complex functions in the future.

An interesting direction of future work is to consider two families of resets, each having their own individual timers. For example, recent work has shown that the time interval between two proteins burst events follows non-exponential statistical distributions [57]. Thus, by having a second timer we can capture memory in timing of burst events. Another direction of future work is to expand this analysis to multi-mode SHS allowing for timer-based switching between stochastic dynamical systems.

\section{ACKNOWLEDGMENT}

This work is supported by ARO Grant W911NF1910243.

\section{APPENDIX}

\subsection{Proof of Theorem 1}

Our proof starts from the derivation of the chemical master equation to trace the probabilistic evolution of states of the system. Based on the forward Kolmogorov equation for stochastic hybrid systems, we can solve for the joint probability 
distribution of random variable $\boldsymbol{\tau}$ and the vector of states $\boldsymbol{x}$ at the steady state

$$
\begin{aligned}
& \frac{\partial p(\tau, x)}{\partial \tau}+\frac{\partial}{\partial x}((\hat{a}+A x) p(\tau, x))= \\
& h_{2}(\tau) p\left(\tau, J_{2}^{-1}\left(x-\hat{r}_{2}\right)\right)-h_{2}(\tau) p(\tau, x)-h_{1}(\tau) p(\tau, x),
\end{aligned}
$$

where $\frac{\partial}{\partial x}$ is partial derivative vector defined as

$$
\frac{\partial}{\partial x}=\left[\frac{\partial}{\partial x_{1}}, \frac{\partial}{\partial x_{1}} \ldots \frac{\partial}{\partial x_{n}}\right]^{\top} .
$$

Now we define conditional moment $\langle\boldsymbol{x} \mid \boldsymbol{\tau}\rangle$ as

$$
\langle\boldsymbol{x} \mid \boldsymbol{\tau}\rangle \equiv\langle\boldsymbol{x} \mid \boldsymbol{\tau}=\tau\rangle=\frac{1}{p(\tau)} \int_{0}^{+\infty} x p(\tau, x) d x,
$$

and we write mean dynamics of $\langle\boldsymbol{x} \mid \boldsymbol{\tau}\rangle$ by taking derivative with respect to $\tau$ in $(47)$

$$
\begin{aligned}
\frac{\partial\langle\boldsymbol{x} \mid \boldsymbol{\tau}\rangle}{\partial \tau} & =-\frac{\frac{\partial p(\tau)}{\partial \tau}}{p(\tau)^{2}} \int_{0}^{+\infty} x p(\tau, x) d x \\
& +\frac{1}{p(\tau)} \int_{0}^{+\infty} x \frac{\partial p(\tau, x)}{\partial \tau} d x
\end{aligned}
$$

By substituting derivative of equation (6) and also (45) into mean dynamics, we will have

$$
\begin{aligned}
\frac{\partial\langle\boldsymbol{x} \mid \boldsymbol{\tau}\rangle}{\partial \tau} & =-\frac{1}{p(\tau)} \int_{0}^{+\infty} x \frac{\partial}{\partial x}((\hat{a}+A x) p(\tau, x)) d x \\
& -\frac{h_{2}(\tau)}{p(\tau)} \int_{0}^{+\infty} x p(\tau, x) d x \\
& +\frac{h_{2}(\tau)}{p(\tau)} \int_{0}^{+\infty} x p\left(\tau, J_{2}^{-1}\left(x-\hat{r}_{2}\right)\right) d x
\end{aligned}
$$

After Applying some mathematical steps, (49) is simplified as

$$
\frac{\partial\langle\boldsymbol{x} \mid \boldsymbol{\tau}\rangle}{\partial \tau}=\left(A+h_{2}(\tau)\left(J_{2}-I_{n}\right)\right)\langle\boldsymbol{x} \mid \boldsymbol{\tau}\rangle+\left(\hat{a}+\hat{r}_{2} h_{2}(\tau)\right) .
$$

Furthermore, conditional second order moment is

$$
\left\langle\boldsymbol{x} \boldsymbol{x}^{\top} \mid \boldsymbol{\tau}\right\rangle \equiv\left\langle\boldsymbol{x} \boldsymbol{x}^{\top} \mid \boldsymbol{\tau}=\tau\right\rangle=\frac{1}{p(\tau)} \int_{0}^{+\infty} x x^{\top} p(\tau, x) d x .
$$

After taking derivative with respect to $\tau$ from both sides of (51) and also using vectorization [58], $\operatorname{vec}\left(\mathrm{A}_{1} \mathrm{~A}_{2} \mathrm{~A}_{3}\right)=\left(\mathrm{A}_{3}^{\top} \otimes \mathrm{A}_{1}\right) \operatorname{vec}\left(\mathrm{A}_{2}\right)$ we will have get $\frac{\partial \operatorname{vec}\left\langle\overline{\boldsymbol{x} \boldsymbol{x}^{\top}|\boldsymbol{\tau}\rangle}\right.}{\partial \tau}$.

\subsection{Cell-cycle time with Gamma distribution}

$$
\begin{gathered}
\left\langle e^{-\gamma \boldsymbol{\tau}_{s}}\right\rangle=\left(C V_{\boldsymbol{\tau}_{s}}^{2} \ln (2)+1\right)^{-\frac{1}{C V_{\boldsymbol{\tau}_{s}}^{2}}} \\
\left\langle\boldsymbol{\tau}_{\boldsymbol{s}} e^{-\gamma \boldsymbol{\tau}_{\boldsymbol{s}}}\right\rangle=\left(C V_{\boldsymbol{\tau}_{s}}^{2} \ln (2)+1\right)^{-\frac{1}{C V_{\boldsymbol{\tau}_{s}}^{2}}-1} \\
\left\langle\boldsymbol{\tau}_{\boldsymbol{s}}{ }^{3}\right\rangle=\left(C V_{\boldsymbol{\tau}_{s}}^{2}+1\right)\left(2 C V_{\boldsymbol{\tau}_{s}}^{2}+1\right)\left\langle\boldsymbol{\tau}_{\boldsymbol{s}}{ }^{3}\right\rangle
\end{gathered}
$$




\section{References}

[1] J. P. Hespanha, "A model for stochastic hybrid systems with application to communication networks," Nonlinear Analysis: Theory, Methods 85 Applications, vol. 62, pp. 1353-1383, 2005.

[2] S. Bohacek, J. P. Hespanha, J. Lee, and K. Obraczka, "A hybrid systems modeling framework for fast and accurate simulation of data communication networks," in Proc. of the ACM Int. Conf. on Measurements and Modeling of Computer Systems (SIGMETRICS), vol. 31, 2003, pp. 58-69.

[3] J. Hu, "Application of stochastic hybrid systems in power management of streaming data," in Proc. of the 2006 Amer. Control Conference, Minneapolis, MN, 2006, pp. 4754-4759.

[4] M. Strelec, K. Macek, and A. Abate, "Modeling and simulation of a microgrid as a stochastic hybrid system," in 3rd IEEE PES International Conference and Exhibition on Innovative Smart Grid Technologies (ISGT Europe), 2012, pp. 1-9.

[5] A. David, D. Du, K. Larsen, M. Mikučionis, and A. Skou, "An evaluation framework for energy aware buildings using statistical model checking," Science China Information Sciences, vol. 55, pp. 2694-2707, 2012.

[6] A. Visintini, W. Glover, J. Lygeros, and J. Maciejowski, "Monte carlo optimization for conflict resolution in air traffic control," IEEE Transactions on Intelligent Transportation Systems, vol. 7, pp. 470-482, 2006.

[7] W. Liu and I. Hwang, "Stochastic hybrid system model with applications to aircraft trajectory prediction and conflict detection," AIAA Journal on Guidance, Dynamics and Control, vol. 34, 2011.

[8] J. Hu and M. Prandini, "Aircraft conflict detection: A method for computing the probability of conflict based on markov chain approximation," Proc. of the 7th European Control Conference, Cambridge, United Kingdom, pp. 2225-2230, 2003.

[9] A. Julius and G. Pappas, "Approximations of stochastic hybrid systems," IEEE Transactions on Automatic Control, vol. 54, pp. 1193-1203, 2009.

[10] E. Buckwar and M. Riedler, "An exact stochastic hybrid model of excitable membranes including spatio-temporal evolution," Journal of Mathematical Biology, vol. 63, pp. 1051-1093, 2011.

[11] A. Singh and J. P. Hespanha, "Stochastic hybrid systems for studying biochemical processes," Philosophical Transactions of the Royal Society A, vol. 368, pp. 4995-5011, 2010.

[12] J. H. Matis and T. R. Kiffe, "On apprroximating the moments of the equilibrium distribution of a stochastic logisitc model," Biometrics, vol. 52, pp. 155-166, 1996. 
[13] D. Antunes and A. Singh, "Quantifying gene expression variability arising from randomness in cell division times," Journal of Mathematical Biology, vol. 71, pp. 437-463, 2015.

[14] C. A. Vargas-García, M. Soltani, and A. Singh, "Conditions for cell size homeostasis: A stochastic hybrid systems approach," IEEE Life Sciences Letters, vol. 2, pp. 47-50, 2016.

[15] D. Riley, X. Koutsoukos, and K. Riley, "Modelling and analysis of the sugar cataract development process using stochastic hybrid systems," IET Systems Biology, vol. 3, pp. 137-154, 2009.

[16] A. Singh, "Noise mechanisms in synaptic transmission and their impact on spike-timing precision," IEEE 56th Annual Conference on Decision and Control (CDC), 2017.

[17] J. Hu, J. Lygeros, and S. Sastry, "Modeling subtilin production in bacillus subtilis using stochastic hybrid systems," in Hybrid Systems: Computation and Control. Springer, 2004, pp. 417-431.

[18] Z. Vahdat, Z. Xu, and A. Singh, "Modeling and characterization of neuronal synapses using stochastic hybrid systems," IEEE 58th Annual Conference on Decision and Control (CDC), 2019.

[19] M. Soltani and A. Singh, "Moment-based analysis of stochastic hybrid systems with renewal transitions," Automatica, vol. 84, pp. 62-69, 2017.

[20] D. Antunes, J. P. Hespanha, and C. Silvestre, "Stochastic hybrid systems with renewal transitions: Moment analysis with application to networked control systems with delays," SIAM Journal on Control and Optimization, vol. 51, pp. 1481-1499, 2013.

[21] D. Antunes, J. Hespanha, and C. Silvestre, "Stability of networked control systems with asynchronous renewal links: An impulsive systems approach," Automatica, vol. 49, pp. 402-413, 2013.

[22] J. P. Hespanha, "Modeling and analysis of networked control systems using stochastic hybrid systems," Annual Reviews in Control, vol. 38, pp. 155$170,2014$.

[23] M. Soltani and A. Singh, "Control design and analysis of a stochastic eventdriven system," IEEE Conference on Decision and Control (CDC), pp. 5789-5794, 2018.

[24] D. M. Suter, N. Molina, D. Gatfield, K. Schneider, U. Schibler, and F. Naef, "Mammalian genes are transcribed with widely different bursting kinetics," Science, vol. 332, pp. 472-474, 2011.

[25] R. D. Dar, B. S. Razooky, A. Singh, T. V. Trimeloni, J. M. McCollum, C. D. Cox, M. L. Simpson, and L. S. Weinberger, "Transcriptional burst 
frequency and burst size are equally modulated across the human genome," Proceedings of the National Academy of Sciences, vol. 109, pp. $17454-$ $17459,2012$.

[26] I. Golding, J. Paulsson, S. Zawilski, and E. Cox, "Real-time kinetics of gene activity in individual bacteria," Cell, vol. 123, pp. 1025-1036, 2005.

[27] J. R. Chubb, T. Trcek, S. M. Shenoy, and R. H. Singer, "Transcriptional pulsing of a developmental gene," Current Biology, vol. 16, pp. 1018-1025, 2006.

[28] T. Trcek, J. A. Chao, D. R. Larson, H. Y. Park, D. Zenklusen, S. M. Shenoy, and R. H. Singer, "Single-mRNA counting using fluorescent in situ hybridization in budding yeast," Nature Protocols, vol. 7, pp. 408-419, 2012.

[29] N. Molina, D. M. Suter, R. Cannavo, B. Zoller, I. Gotic, and F. Naef, "Stimulus-induced modulation of transcriptional bursting in a single mammalian gene," Proceedings of the National Academy of Sciences, 2013.

[30] M. J. Dunlop, R. S. Cox, J. H. Levine, R. M. Murray, and M. B. Elowitz, "Regulatory activity revealed by dynamic correlations in gene expression noise," Nature Genetics, vol. 40, pp. 1493-1498, 2008.

[31] A. Raj, C. Peskin, D. Tranchina, D. Vargas, and S. Tyagi, "Stochastic mRNA synthesis in mammalian cells," PLOS Biology, vol. 4, p. e309, 2006.

[32] A. Milias-Argeitis, S. Summers, J. Stewart-Ornstein, I. Zuleta, D. Pincus, H. El-Samad, M. Khammash, and J. Lygeros, "In silico feedback for in vivo regulation of a gene expression circuit," Nature Biotechnology, vol. 29, pp. 1114-1116, 2011.

[33] S. Keskin, G. S. Devakanmalai, S. B. Kwon, A. Singh, A. Ay, and E. M. Ozbudak, "Notch signaling boosts transcription burst frequency to tame noise in the vertebrate segmentation clock," Cell Reports, vol. 23, pp. 2175$2185,2018$.

[34] A. Eldar and M. B. Elowitz, "Functional roles for noise in genetic circuits," Nature, vol. 467, pp. 167-173, 2010.

[35] M. Kærn, T. C. Elston, W. J. Blake, and J. J. Collins, "Stochasticity in gene expression: from theories to phenotypes," Nature Reviews Genetics, vol. 6 , pp. 451-464, 2005.

[36] G. Balázsi, A. van Oudenaarden, and J. J. Collins, "Cellular decision making and biological noise: From microbes to mammals," Cell, vol. 144, pp. 910-925, 2014.

[37] E. Abranches, A. M. V. Guedes, M. Moravec, H. Maamar, P. Svoboda, A. Raj, and D. Henrique, "Stochastic nanog fluctuations allow mouse embryonic stem cells to explore pluripotency," Development, vol. 141, pp. 2770-2779, 2014. 
[38] A. Raj and A. van Oudenaarden, "Nature, nurture, or chance: stochastic gene expression and its consequences," Cell, vol. 135, pp. 216-226, 2008.

[39] S. M. Shaffer, M. C. Dunagin, S. R. Torborg, E. A. Torre, B. Emert, C. Krepler, M. Beqiri, K. Sproesser, P. A. Brafford, M. Xiao et al., "Rare cell variability and drug-induced reprogramming as a mode of cancer drug resistance," Nature, vol. 546, pp. 431-435, 2017.

[40] J. Paulsson, O. G. Berg, and M. Ehrenberg, "Stochastic focusing: Fluctuation-enhanced sensitivity of intracellular regulation," Proceedings of the National Academy of Sciences, vol. 97, pp. 7148-7153, 2000.

[41] B. Munsky, B. Trinh, and M. Khammash, "Listening to the noise: random fluctuations reveal gene network parameters," Molecular Systems Biology, vol. 5, p. 318, 2009.

[42] M. Soltani and A. Singh, "Moment analysis of linear time-varying dynamical systems with renewal transitions," SIAM Journal on Control and Optimization, vol. 57, no. 4, pp. 2660-2685, 2019.

[43] M. Soltani, C. A. Vargas-Garcia, D. Antunes, and A. Singh, "Intercellular variability in protein levels from stochastic expression and noisy cell cycle processes," PLOS Computational Biology, p. e1004972, 2016.

[44] D. Huh and J. Paulsson, "Random partitioning of molecules at cell division," Proceedings of the National Academy of Sciences, vol. 108, no. 36, pp. $15004-15009,2011$.

[45] — , "Non-genetic heterogeneity from stochastic partitioning at cell division," Nature Genetics, vol. 43, pp. 95-100, 2011.

[46] T. Fukaya, B. Lim, and M. Levine, "Enhancer control of transcriptional bursting," Cell, vol. 166, pp. 358-368, 2015.

[47] C. R. Bartman, S. C. Hsu, C. C.-S. Hsiung, A. Raj, and G. A. Blobel, "Enhancer regulation of transcriptional bursting parameters revealed by forced chromatin looping," Molecular Cell, vol. 62, pp. 237 - 247, 2016.

[48] A. M. Corrigan, E. Tunnacliffe, D. Cannon, and J. R. Chubb, "A continuum model of transcriptional bursting," eLife, vol. 5, p. e13051, 2016.

[49] S. Chong, C. Chen, H. Ge, and X. S. Xie, "Mechanism of transcriptional bursting in bacteria," Cell, vol. 158, pp. 314-326, 2014.

[50] A. Singh, B. Razooky, C. D. Cox, M. L. Simpson, and L. S. Weinberger, "Transcriptional bursting from the HIV-1 promoter is a significant source of stochastic noise in HIV-1 gene expression," Biophysical Journal, vol. 98, pp. L32-L34, 2010.

[51] J. M. Pedraza and J. Paulsson, "Effects of molecular memory and bursting on fluctuations in gene expression," Science, vol. 319, pp. $339-343,2008$. 
[52] T. Jia and R. V. Kulkarni, "Intrinsic noise in stochastic models of gene expression with molecular memory and bursting," Journal of Mathematical Biology, vol. 106, p. 058102, 2011.

[53] N. Kumar, A. Singh, and R. V. Kulkarni, "Transcriptional bursting in gene expression: analytical results for general stochastic models," PLOS Computational Biology, vol. 11, p. e1004292, 2015.

[54] P. Bokes and A. Singh, "Gene expression noise is affected deferentially by feedback in burst frequency and burst size," Journal of Mathematical Biology, vol. 74, pp. 1483-1509, 2017.

[55] V. Shahrezaei and P. S. Swain, "Analytical distributions for stochastic gene expression," Proceedings of the National Academy of Sciences, vol. 105, pp. $17256-17261,2008$.

[56] N. Friedman, L. Cai, and X. Xie, "Linking stochastic dynamics to population distribution: an analytical framework of gene expression," Physical Review Letters, vol. 97, p. 168302, 2006.

[57] B. Daigle, M. Soltani, L. Petzold, and A. Singh, "Inferring single-cell gene expression mechanisms using stochastic simulation," Bioinformatics, vol. 31, pp. 1428-1435, 2015.

[58] H. D. Macedo and J. N. Oliveira, "Typing linear algebra: A biproductoriented approach," Science of Computer Programming, vol. 78, no. 11, pp. 2160-2191, 2013. 